\title{
Research on AC/DC Distribution Network Planning Method
}

\author{
Ende $\mathrm{Hu}^{1 *}$, Shuai Wang ${ }^{2}$, Wei Song ${ }^{1}$, Haibo Zhao ${ }^{1}$, Li Chunhui ${ }^{1}$, Liang Tian, Zhuo Liu Zeyuan Shen ${ }^{1}$ and Xing Yahong ${ }^{1}$ \\ ${ }^{1}$ Economic and Technical Research Institute of SEPC of SGCC, Taiyuan, China \\ ${ }^{2}$ Electric Power Research Institute of SEPC of SGCC, Taiyuan, China
}

\begin{abstract}
The transition from traditional $\mathrm{AC}$ distribution system to future mature $\mathrm{AC} / \mathrm{DC}$ hybrid distribution mode is an effective means to solve the challenges of high penetration of distributed energy, diversification of energy types, high requirements of power quality and power supply reliability.AC / DC hybrid distribution system can be used in many scenarios by virtue of its technical advantages. This paper analyzes the typical application scenarios of AC / DC distribution network from the perspective of "source network load". Combined with the research status of AC / DC hybrid distribution network, this paper introduces the planning method from three aspects: paper, standard and typical algorithm. Finally, a demonstration project is taken as an example to illustrate the key points of the planning.
\end{abstract}

\section{Introduction}

Distribution network is an important platform to carry all kinds of users, such as distributed generation, AC/DC load and energy storage[1]. It is the key link to promote the construction of smart grid and solve the energy crisis. Different power supply areas have different energy types and user access requirements, and the penetration of new energy is high[2]. Specific users, especially highprecision electronic industry, have high requirements on power supply reliability and power quality[3]. The transition from traditional $\mathrm{AC}$ distribution system to future mature AC/DC hybrid distribution mode is a feasible way to solve the problems and challenges faced by the current distribution network[5]. Compared with AC distribution network, AC/DC distribution network has the characteristics of high transmission efficiency, large transmission capacity, long transmission distance, high power supply reliability and flexible power supply, which can meet the access requirements of various AC and DC users[6].

The development of traditional AC distribution network planning technology is relatively mature. At present, the research on AC/DC distribution system planning mainly focuses on DC distribution voltage level, grid structure, wiring mode, grounding mode and key equipment. This paper mainly introduces the application scenarios, planning algorithms and examples of $\mathrm{AC}$ and DC distribution network, summarizes the common application scenarios, summarizes the previous planning algorithms, and finally compares two distribution network planning schemes under the same conditions.

\section{Application scenarios of AC/DC distribution network}

The research on the application scenarios of $\mathrm{AC} / \mathrm{DC}$ distribution system mainly focuses on the demonstration applications of industrial power supply, residential building power supply, AC partition interconnection, distributed power supply, data center power supply, etc. The typical application scenarios of urban DC distribution network can be divided from the perspectives of power supply, load classification and distribution and distribution network development form in the scene, including three major scenarios: Service of new energy grid connection, service distribution network interconnection and service DC load power supply. The grid connection of service new energy is divided into two small scenarios: centralized new energy power generation and centralized decentralized new energy power generation; service distribution network interconnection is divided into two types of small scenarios: interconnection of different voltage levels and interconnection of different supply areas of the same voltage level; power consumption of DC load is divided into DC load concentration area, industrial park, urban commercial and residential area and $\mathrm{AC}$ distribution network with limited capacity Four kinds of small scenes of urban load center.

At present, the project application scenarios from the "source grid load" level mainly include three types: serving for new energy grid connection, serving for distribution network interconnection, and serving for DC load power consumption. These three types cover the main scenarios of distribution network construction; AC/DC distribution network project construction can not be limited to a single application scenario, and the

"Corresponding author: 1551865032@163.com 
diversified future distribution network should meet the distribution of multiple scenarios Net combination.

\section{Papers and Standardization of distribution network}

\subsection{Summary of papers on AC/DC distribution network planning}

There are many ways to construct AC/DC hybrid distribution network. One is to transform part of the existing AC lines into DC lines and connect them with $\mathrm{AC}$ lines through converters to form AC/DC hybrid distribution networks; the other is to divide the planning of $\mathrm{AC} / \mathrm{DC}$ distribution network into two types: considering distributed generation and not considering distributed generation. To minimize the total investment and operation cost of the expansion planning of $\mathrm{AC} / \mathrm{DC}$ distribution network with distributed generation as the objective, this paper gives the optimization planning scheme of adding lines in the original distribution network and distributing the distribution points of collaborative distributed generation.

The paper[7] proposed a source grid coordinated medium voltage $\mathrm{AC} / \mathrm{DC}$ hybrid distribution network converter location method. Through the location of the converter, the $\mathrm{AC} / \mathrm{DC}$ division of the distribution network is determined at the network end, and the capacity allocation of photovoltaic and battery energy storage at each access point is determined at the source end.

The paper[8] takes the net income of the medium voltage AC/DC hybrid distribution network equipment in the whole life cycle including construction and operation as the objective function, and uses the optimization model including mixed integer and secondorder cone relaxation to solve the operation income and cost of different location schemes; adopts the genetic algorithm based on elite strategy to optimize the objective function, and then determines the optimal location scheme. In this paper, fan Anjie proposed a bilevel programming model of "upper level planning and lower level operation". The upper level determined the DC distribution points and AC/DC network architecture with the minimum comprehensive annual cost as the goal, and the lower layer as the operation layer to obtain the optimal operation scenario of flexible resources.

The paper[9] proposes a planning method considering DC transformation and expansion of grid structure, and establishes a DC / AC hybrid distribution network planning model with DG based on time series multi scenario. In this model, the configuration of distributed generation, the grid structure to be transformed by DC, the access location of expanded grid and whether to connect in DC form are taken as decision variables. The objective function considers the investment of DG and grid, converter investment of DG and load, network loss and voltage stability.

The paper[10] proposes a new network configuration planning model for AC/DC hybrid distribution network. The model determines the optimal configuration of branches and buses in $\mathrm{AC} / \mathrm{DC}$ hybrid distribution network. By using Monte Carlo simulation technology, the network configuration planning problem is divided into two nested optimization problems to obtain the optimal solution: the main problem takes the system construction cost and operation cost as the objective function, and uses genetic algorithm to obtain the optimal AC/DC hybrid configuration; the sub problem is refined by economic dispatch The operation cost of the main problem is calculated to determine the optimal power flow solution of each configuration obtained by genetic algorithm.

\subsection{Standardization of AC/DC distribution system planning and design}

The technical standards related to $\mathrm{AC}$ distribution network planning and design are relatively perfect. The standardization of $\mathrm{AC} / \mathrm{DC}$ distribution system planning and design mainly focuses on DC distribution voltage level, AC/DC distribution network interconnection, network structure, evaluation method, etc.

China Electric Power Enterprise Federation issued the "DC distribution voltage" (T/CEC 107-2016), which specifies the preferred value, alternative value, DC distribution transmission capacity and other aspects of the nominal voltage of high and medium voltage DC distribution system. China National Standardization Administration issued the guidelines for medium and low voltage DC distribution voltage (GB/T 35727-2017), which specifies the preferred value and alternative value of nominal voltage and DC voltage deviation of medium and low voltage DC distribution system. China Electric Power Enterprise Federation issued technical requirements for interconnection of $\mathrm{DC}$ distribution network and AC distribution network (T/CEC) 1672018), which specifies the AC/DC interconnection mode, $\mathrm{AC} / \mathrm{DC}$ interconnection voltage, $\mathrm{AC} / \mathrm{DC}$ interconnection device, reactive power configuration, protection coordination, grounding and other technical requirements involved in the interconnection technology between DC distribution network and AC distribution network, providing technical principle support for the construction and development of $\mathrm{AC} / \mathrm{DC}$ distribution system. On the same day, technical guidelines for typical grid structure and power supply scheme of medium voltage DC distribution network (T/CEC) were issued 166-2018), the topological structures of different typical grid structures, such as double ended structure and single ended ring structure, are classified, and the technical requirements and typical power supply schemes of power supply are given for reference. Finally, power supply schemes are proposed and specified for several typical application scenarios of medium voltage DC distribution, such as centralized load, decentralized load, renewable energy collection and high reliability requirements Applicable grid topology, connection type, grounding mode, expansion mode, etc. State Grid Corporation of China issued the guidelines for planning and design of $\mathrm{AC} / \mathrm{DC}$ hybrid distribution network, which specifies the relevant technical principles of $\mathrm{AC} / \mathrm{DC}$ 
hybrid distribution network in terms of voltage level, grid structure, main equipment, system secondary, power supply and user access. It is pointed out that AC power supply is preferred for high-voltage $\mathrm{AC} / \mathrm{DC}$ hybrid distribution network, and DC power supply can be used for power island and island power grid with large capacity or long-distance power transmission demand. Distributed generation, DC load, sensitive load, energy storage and other power supply areas with large demand can adopt DC based power supply mode. On the same day, the guidelines for comprehensive evaluation of AC/DC hybrid distribution networks were issued The three-level comprehensive evaluation index system structure of $\mathrm{AC} / \mathrm{DC}$ hybrid distribution network is specified, and the specific evaluation index content and calculation method are given, which provides evaluation basis and method for the planning scheme of $\mathrm{AC} / \mathrm{DC}$ hybrid distribution network within the jurisdiction of the company. The evaluation results can guide the planning and construction of $\mathrm{AC} / \mathrm{DC}$ hybrid distribution network, guide the development direction of $\mathrm{AC} / \mathrm{DC}$ hybrid distribution network and assist AC/DC hybrid distribution network Scientific development of DC hybrid distribution network.

\subsection{AC/DC distribution network planning algorithm}

Integrated resource planning (IRP) is a kind of resource optimization which takes the power supply side resources and power demand side resources as the overall optimization objects. The core idea of integrated resource planning is to divide the response of power supply side and demand side into power system planning, analyze the cost-effectiveness of power supply scheme and energy-saving scheme, and form a comprehensive planning scheme which benefits the society, power enterprises (companies) and users and can meet the future power demand. Through power demand side management, more reasonable and effective use of energy resources, increase environmental protection, reduce power construction investment, reduce power grid operation expenditure, provide users with the most economic energy resources services.

Optimization method, also known as operational research method, mainly uses mathematical methods to study the optimization approaches and schemes of various systems, so as to provide scientific basis for decision-makers. The main research object of optimization method is the management problems of various organized systems and their production and operation activities. The purpose of the optimization method is to find a reasonable use of human, material and financial resources of the optimal scheme, play and improve the efficiency and efficiency of the system, and ultimately achieve the optimal goal of the system. It includes linear optimization method, optimization method with or without constraints, intelligent algorithm, etc. In this project, the principle and method of optimization is the main means to solve the planning scheme in typical scenarios, and it is an important mathematical tool of the project.

The traditional heuristic method is based on intuitive analysis and combined with the experience of planners. Compared with the classical mathematical method, it can simulate the actual power behavior more accurately. It is usually based on the sensitivity of a certain performance index of the system to the line parameters on the feasible path, and the iteration is carried out step by step according to certain principles until a scheme meeting the requirements is obtained. Modern heuristic methods include genetic algorithm, evolutionary programming, simulated annealing, tabu search, ant colony optimization, particle swarm optimization, artificial neural network and so on. The characteristic of heuristic algorithm is to consider two indexes of planning efficiency and planning effect. In practice, many heuristic methods, especially modern heuristic methods, can often give satisfactory solutions. The advantages of heuristic method are intuitionistic, flexible and fast in calculation. It is convenient for planners to participate in specific decision-making in the planning process.

\section{Comparison of planning schemes}

Taking the planning scheme of a demonstration project as an example, the DC load of the project is $1.8 \mathrm{mw}$, the AC load is $0.7 \mathrm{mw}$, and the DC load is mainly server; AC load includes air conditioning system, heating system, lighting system and communication related equipment.

The scheme 1 is fully functional. At the same time, the performance of multi module power electronic devices in DC distribution network is verified and analyzed. Moreover, direct access to new energy can fully verify the performance of flexible substation. Second, it is innovative and exemplary. The centralized photovoltaic DC / DC converter station itself has a certain degree of innovation, which is the current research hotspot. Third, the economy is good. The construction cost of flexible substation and DC / AC module is lower than that of PV station. The photovoltaic DC / DC converter station is about $3.5 \mathrm{~km}$ away from the small two flexible substations, and the line investment is saved.

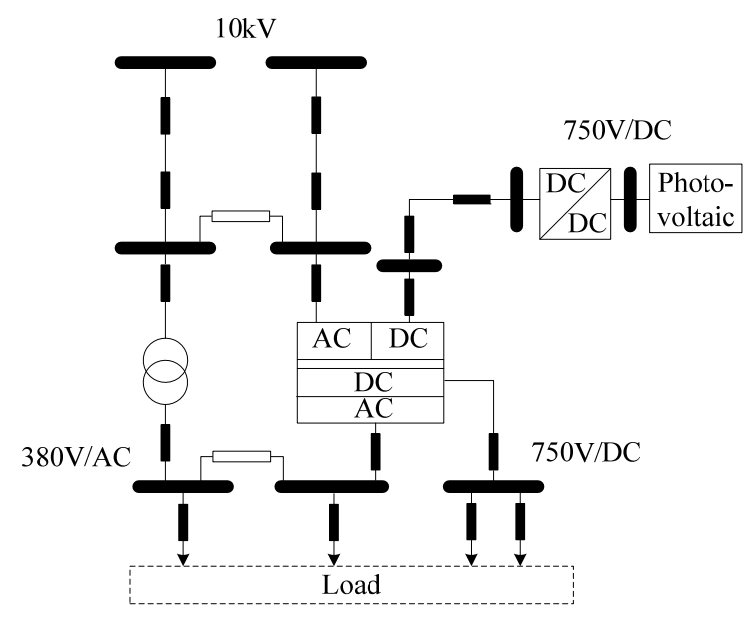

Figure 1. Electric wiring diagram of scheme 1 
In the scheme 2, through the construction of $\mathrm{AC} / \mathrm{DC}$ rectifier station and flexible substation, a complete $\mathrm{AC} / \mathrm{DC}$ distribution network with two terminal topology is constructed, which meets the verification requirements of flexible substation. The uncertainty factors are relatively small, which can reduce the risk of flexible substation research and development difficulty, high equipment cost and great influence of reliability on the system.

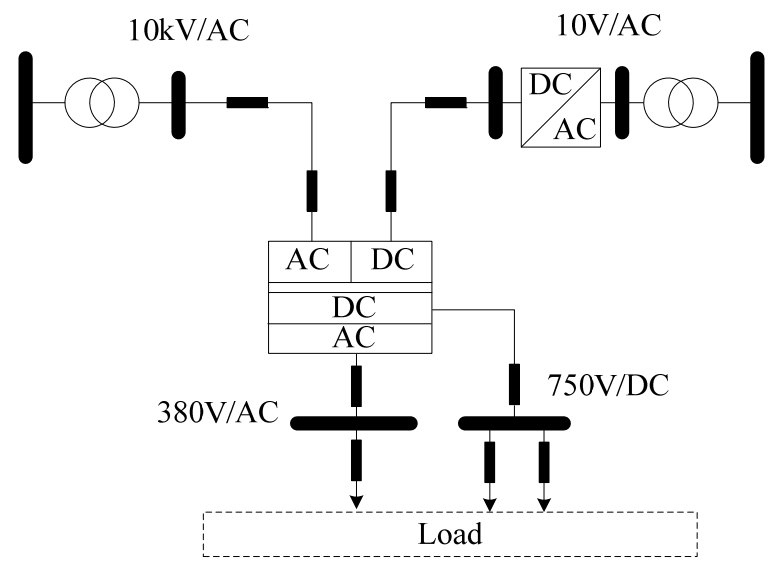

Figure 2. .Electric wiring diagram of scheme 2

Scheme 3 uses the spare $10 \mathrm{kV}$ Bay of conventional substation, and realizes the operation verification of \pm $10 \mathrm{kV}$ port function of flexible substation through relatively simple topology. It has simple structure, little influence on the original communication system, controllable construction sequence and convenient operation and maintenance. Xiaoertai $35 \mathrm{kV}$ station has no spare $10 \mathrm{kV}$ interval, so cloud computing $110 \mathrm{kV}$ substation is selected as the $10 \mathrm{kV}$ AC side power supply of AC/DC. The station is located near Alibaba Zhangbei innovation $\mathrm{R} \& \mathrm{D}$ Exhibition Center, with short line length.

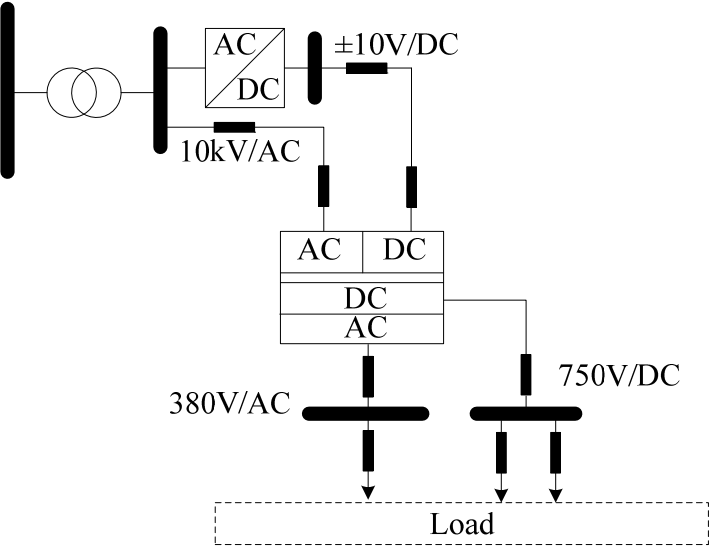

Figure 3. Electric wiring diagram of scheme 3

Table 1. Cost of three schemes

\begin{tabular}{cc}
\hline Scheme & Cost \\
\hline Case1 & 6161 \\
Case2 & 6586 \\
Case3 & 5686 \\
\hline
\end{tabular}

The scheme 1 can not only meet the construction objectives of the project, but also take into account the verification needs of new technologies. It has four highlights, namely, scientific and technological innovation, clean energy consumption, diversified power supply of emerging industries, and photovoltaic poverty alleviation services. It is innovative, demonstrative and economical.

\section{Conclusion}

In the planning, we should strengthen the economic research of AC/DC distribution network, deeply understand the development status and trend of new energy and DC load, consider the distribution characteristics of new energy and DC load, formulate differentiated construction mode according to local conditions, and actively guide the reasonable layout and orderly development of AC/DC distribution network.

At the same time, it analyzes the influencing factors and requirements of $\mathrm{AC} / \mathrm{DC}$ distribution network planning standardization, establishes typical unit module of AC/DC distribution network standardization planning, studies the application selection principle of typical unit module of AC/DC distribution network, studies the regional differentiation application conditions of DC distribution network equipment, and studies the technical principles of DC distribution network equipment standardization application.

There are many kinds of application scenarios for AC/DC distribution system, and the electrical characteristics of large-scale multi energy and multi load access are complex. In different application scenarios, users have different requirements on power supply capacity, power quality and reliability of the system, which results in the existence of system voltage level, grid structure, grounding mode, wiring mode and configuration mode of primary and secondary equipment with traditional AC system Significant difference. Therefore, considering the demand of multi energy integration and supply-demand interaction, the electrical characteristics of large-scale AC / DC user access are analyzed, the basis and technical principles of application scenario division are clarified, and the AC / DC distribution system planning scheme suitable for various typical scenarios is constructed,

\section{References}

1. Ma Zhao, Zhou Xiaoxin, Shang Yuwei, et al. Form and development trend of future distribution system[J]. Proceedings of the CSEE, 2015, 35(6): 1289-129.

2. Jia L, Zhu Y, Wang Y. Architecture design for new AC-DC hybrid micro -grid [A]. IEEE First International Conference on Dc Microgrids [C]. Atlanta: IEEE, 2015.113-118.

3. Kong Li, Pei Wei, Ye Hua, et al. Review of pattern control and stability for hybrid AC/DC distribution power systems[J]. Advanced 
Technology of Electrical Engineering and Energy, 2017, 36(9): 1-10.

4. Sun Guomen, Qi Chen, Han Bei, et al. Research of key technology of $\mathrm{AC} / \mathrm{DC}$ hybrid distribution network planning and operation[J]. Distribution \& Utilization, 2016, 33(8): 717

5. FENG W, LE TUAN A, TJERNBERG L B, et al. A new approach for benefit evaluation of multiterminal VSC-HVDC using a proposed mixed AC/DC optimal power flow[J]. IEEE Transactions. Power Delivery, 2014, 29(1): 432-443.

6. Geng Qi, Hu Yan, He Jianzong, et al. Optimal power flow for $\mathrm{AC}$ and $\mathrm{DC}$ grids based on power electronic transformer[J]. Power System Technology, 2019, 43(9): 3288-3298

7. Wang Wei. Research on optimal planning and typical application scenarios of AC/DC distribution network [D]. Beijing Jiaotong University, 2018

8. Su Jian, Wang Zhuo, Cui Yanyan, Wei Tao, Zhang Wei, Hui Hui, Zhao Mingxin. Research on converter location of medium voltage AC / DC hybrid distribution network based on source grid collaboration [J]. Power supply and consumption, 2019,36 (05): 59-65

9. Hu Xiaobo. Planning of AC / DC hybrid distribution network with distributed energy [D]. North China Electric Power University, 2017

10. Ali G, Mahmoud R H, Sayyed M L. Comprehensive approach for hybrid AC/DC distribution network planning using genetic algorithm[J] IET Generation, Transmission \& Distribution, 2017, 11(16): 38923902. 